International Journal of Clinical \& Medical Allergy (IJCMA)

ISSN 2332-2799

\title{
Social Stigma, Discrimination, Marginalization and Prejudice towards Persons with Tuberculosis: A Systematic Review Study
}

\author{
Case Report
}

Manzoor $\mathrm{S}^{1}$, Zahoor A Ganie ${ }^{2 *}$

${ }^{1}$ Assistant Professor, Department of Social Work, University of Kashmir, Hazratbal, Jammu and Kashmir, India.

${ }^{2}$ Research Scholar, Department of Social Work, University of Kashmir, Jammu and Kashmir, India.

\begin{abstract}
Tuberculosis is one of the most commonly diseases in India. It is a highly infectious disease and most commonly affects the lungs of a person. Tuberculosis (TB) is an infectious disease caused by a Bacterium, Mycobacterium tuberculosis. It is spread through the air by a person suffering from TB. A single patient can infect 10 or more people in a year. India has a long and distinguished tradition of research in TB. Evidence indicates that TB has plagued mankind since ancient times. According to data from WHO on global drug resistance, an estimated 3.6 percent of global incident (new) TB cases, or a total of 440,000 cases, were MDR TB in 2008 (95 percent confidence interval, 390,000-510,000) [1]. The current tuberculosis (TB) control strategy in India largely ignores psychosocial needs of the patients. The present study was prompted by the recognition that, if TB treatment is to be culturally sensitive and effective, the psychosocial problems and issues need to be recognized and addressed. Social stigma, discrimination, marginalization and prejudice are complex problems in the lives of persons with Tuberculosis in India. The diversity and depth of research work underscores the complexity of the linkages between stigma, prejudice and health issues. The development of practical interventions and policies to reduce and attenuate the impacts of stigma and prejudice on health for marginalized populations depends on more fully understanding these linkages.
\end{abstract}

Keywords: Tuberculosis; Stigma; Prejudice; Discrimination and Marginalization.

\section{*Corresponding Author:}

Zahoor Ahmad Ganie,

Research Scholar, Department of Social Work, University of Kashmir, Jammu and Kashmir, India.

E-mail: zahoor_1057@yahoo.com

Received: April 13, 2015

Accepted: May 28, 2015

Published: June 16, 2015

Citation: Manzoor S, Zahoor A Ganie (2015) Social Stigma, Discrimination, Marginalization and Prejudice towards Persons with Tuberculosis: A Systematic Review Study. Int J Clin Med Allergy. 03(2), 14-18. doi: http:/ / dx.doi.org/10.19070/2332-2799-150005

Copyright: Zahoor A Ganie ${ }^{\odot} 2015$. This is an open-access article distributed under the terms of the Creative Commons Attribution License, which permits unrestricted use, distribution and reproduction in any medium, provided the original author and source are credited.

\section{Tuberculosis: A Communicable Disease}

Tuberculosis is a communicable disease by the bacteria Mycobacterium tuberculosis. This disease mostly affects the lungs of a patient. This disease can be transmitted from one person to other person via droplets from the throat and lungs of people with active respiratory infections. The common symptoms of active TB are coughing, sometimes with sputum or blood, chest pain, weight loss, general weakness, fever and night time sweats. TB treatment includes long term medication with psychosocial interventions to main optimal level of health and wellbeing [2]. Studies from the Tuberculosis Research Centre in Chennai and the National Tuberculosis Institute in Bangalore provided key knowledge to improve treatment of TB patients all around the world. Modern anti-TB treatment can cure virtually all patients. It is, however, very important that treatment be taken for the prescribed duration, which in every case is a minimum of 6 months. Because treatment is of such a long duration and patients feel better after just 1-2 months and because many TB patients face other problems such as poverty, unemployment, Discrimination, Marginalization, Stigma, Prejudice and treatment is often interrupted. Therefore, just providing anti-TB medication is not sufficient to ensure that patients are cured. The DOTS strategy ensures that infectious TB patients are diagnosed and treated effectively till cure, by ensuring availability of the full course of drugs and a system for monitoring patient compliance to the treatment. Controlling TB in India is a tremendous challenge. The TB burden in India is still staggering. Every year, 1.8 million people develop the disease, of which about 800,000 are infectious; and until recently 370,000 died of it annually and 1000 every day. The disease is a major barrier to social and economic development. An estimated 100 million workdays are lost due to illness. According to data from WHO on global drug resistance, an estimated 3.6 percent of global incident (new) TB cases, or a total of 440,000 cases, were MDR TB in 2008 (95 percent confidence interval, 390,000-510,000) [3]. The available data on drug-resistant TB are inadequate, however, and lead to an underestimation of the true global burden of MDR TB. In many developing countries where the MDR TB burden is likely to be 
significant, surveillance systems do not exist or lack the capacity to generate reliable data. Even the most recent global surveillance data on MDR TB do not include 79 countries 41 percent of all countries in the world (WHO, 2010). The diagnosis of TB is no longer a death warrant (KrishanLal, President, INSA), but the existence of treatments raises sociological and psychological issues. Patients may take a treatment just until they feel well, which can foster the development of resistance and lead to the spread of the disease. The lack of quick, accurate and inexpensive tests for drug-resistant TB hampers treatment. Drug-resistant TB needs to be diagnosed earlier and with greater specificity than is currently the case, especially given the much greater costs of treating drugresistant TB. India accounts for approximately one-fifth of the global incidence of TB [4]. Fully 40 percent of the country's population is infected with the tubercule bacillus. Each year the country sees 2 million new cases (the global incidence is 9.4 million), which lead to 280,000 deaths annually, although the prevalence of HIV among new cases in India is just 6.4 percent compared with a global average of 12 percent. TB is one of the leading causes of death among adults in India, and it also takes a large toll on the country's younger generation, which makes up a significant proportion of the total population. TB also takes a disproportionately large toll among young females: more than 50 percent of TB cases among females occur before age 34 , and an estimated 100,000 women are rejected by their families every year because they have the disease. Some researchers noted that national-level; all-India studies evaluating the effect of a TB diagnosis on family dynamics could provide more specific data and have an impact on understanding and preventing the rejection of TB patients by their families. TB also disproportionately affects the poorest and most marginalized populations in India, as well as people in their most productive ages 70 percent of TB patients are aged 15-54. People with TB incur an average potential loss of 20-30 percent of their annual household income as a result of 3-4 months of lost work time. In India, about 14 million people fall into poverty each year because they experience unaffordable health care costs, and TB is a major cause of health-related impoverishment. The United Nations' Millennium Development Goals call for halting and beginning to reverse the incidence of TB by 2015. The STOP TB Partnership has established the target of reducing the global burden of TB (defined by per capita prevalence and death rates) by 50 percent relative to 1990 levels by 2015 and the long-term goal of reducing the global incidence of active TB to less than 1 case per million populations per year by 2050. India's strategy for working toward these goals is embodied in its Revised National TB Control Program (RNTCP 2007).

\section{Stigma, Discrimination, marginalization and Prej- udice towards TB patients}

Stigma is an attribute or characteristic of a person that is deeply discrediting. This attribute is devalued in a particular context and calls into question the full humanity of the person. Because of this negatively valued attribute, persons are devalued, spoiled or flawed in the eyes of others [5]. Stigma has been described as a dynamic process of devaluation that 'significantly discredits' an individual in the eyes of others. The qualities to which stigma adheres can be quite arbitrary for example, skin color, manner of speaking, or sexual preference. Within particular cultures or settings, certain attributes are seized upon and defined by others as discreditable or unworthy. Emile Durkheim [6] was the first sociologist to explore stigma as a social phenomenon in 1895 and reported that imagine a society of saints, a perfect cloister of exemplary individuals. Crimes or deviance, properly so-called, will there be unknown; but faults, which appear venial to the layman, will there create the same scandal that the ordinary offense does in ordinary -consciousnesses. If then, this society has the power to judge and punish, it will define these acts as criminal (or deviant) and will treat them. Erving Goffman (1963) [7] was one of the great influential sociologists of the $20^{\text {th }}$ Century and according to him stigma is the phenomenon whereby an individual with an attribute which is deeply discredited by his/her society is rejected as a result of the attribute. Stigma is a process by which the reaction of others spoils normal identity. The major cause of Tuberculosis stigma is the perceived risk of transmission from TB-infected individuals to susceptible community members. Depending on geographic region, however, TB is also stigmatized because of its associations with HIV, poverty, low social class, malnutrition, or disreputable behavior. Tuberculosis patients perceive themselves to be at risk for a number of stigma-related social and economic consequences. Because the most common result of Tuberculosis stigma is isolation from other members of the community, TB infection can substantially impact economic opportunities. When an individual dies of TB, fear of TB stigma can lead families to hide the cause of death from other members of the community, even when such information might be useful in targeted TB screening [8]. Similarly, fear of TB stigma can lead infected individuals to hide their TB status from their families. Tuberculosis stigma has a more significant impact on women and poor or less-educated community members, which is especially concerning given that these groups are often at higher risk for health disparities. TB stigma may, therefore, worsen preexisting gender and class based health disparities. Stigma in tuberculosis patients is usually of two types - one i.e. a fear of the patient about other's behavior to him and a sense of inferiority due to development of tuberculosis i.e. perceived stigma; and other due to actual discrimination or being actually avoided by the people since the patient has now tuberculosis i.e. enacted stigma. Patient often tries to hide his/her disease from others due to stigma resulting in further delay in diagnosis and treatment and thus increase chances of transmission to healthy community. Fear of infection had been identified as the main reason for the stigmatization attitudes and behavior of both health professionals and community members towards those with TB. Tuberculosis is reported to have negative social consequences. This includes family members requiring the patient to use separate utensils or eat and sleep in a different room [9] as well as outright avoidance and isolation [10]. Most authors identify the perceived contagiousness of TB as a leading cause of stigmatization [11]. In some studies, women stated their husbands might leave them if they were diagnosed with TB, while those who were not married feared their marriage prospects would decrease [12]. Lack of knowledge regarding routes of TB transmission may also contribute to TB stigma [13]. Even among people with relatively good knowledge of TB transmission and transmissibility, however, the perceived risk of transmission can lead to stigmatization and isolation of individuals with TB. Some TB patients feared losing their jobs [14]. Occasionally, the negative social consequences of TB continued beyond the period of infectiousness, and in some cases beyond completion of treatment [12]. TB stigma also results in a sense of shame or guilt, leading to self-isolation as TB-infected individuals internalize their community's negative judgments about the disease [15]. Fear of being isolated or rejected was reported to cause persons with prolonged cough to try to conceal their disease, withdraw from others, and avoid seeking care for fear of officially being diagnosed as a TB 
patient $[9,10,14]$. The reasons for stigmatizing attitudes vary, but generally fall into three categories: inadequate or incorrect knowledge of TB, association of TB with poverty, poor hygiene, or particular behavior among marginalized populations, and association of TB with the occurrence of HIV/AIDS. Some reports identified fear of infection and transmission as sources of stigma [16]. This was compounded by incorrect knowledge about transmission of TB, including being hereditary [17] or from smoking [18, 19]. Others reported incorrect knowledge of treatment and curability [20] including viewing a TB diagnosis as a death sentence. When isolation and rejection were reported to continue beyond the period of infectiousness, it was due to an incorrect knowledge of treatment and curability and therefore a source of stigma, rather than a public health measure $[14,21]$. Tuberculosis stigma may also exist due to the people, circumstances, or behaviors it is associated with. Some view TB as a dirty disease, and one that is associated with poverty or poor hygiene $[9,18,22]$. The perception of stigma by other people or by the stigmatized person leads to discrimination part of which may be towards oneself, where an individual feels unworthy or guilty, leading to lack of self-worth, depression and abnormal behavior such as self isolation, avoidance behavior and introversion.

When stigma is acted upon, the result is discrimination. Discrimination consists of actions or omissions that are derived from stigma and directed towards those individuals who are stigmatized. Discrimination, as defined by UNAIDS [23] (2000) in the Protocol for Identification of Discrimination Against People Living with HIV and TB, refers to any form of arbitrary distinction, exclusion, or restriction affecting a person, usually but not only by virtue of an inherent personal characteristic or perceived belonging to a particular group in the case of HIV/AIDS and TB. Discrimination means treating some people differently from others. It is not always unlawful, because in occupational setting people will be paid wages depending on their status and skills. However, there are several reasons for which employers can't discriminate against any person based on diseases, religion, caste, sex, region, nationality, color, sexual orientation, race and disability etc. Discrimination should not been done on treatment or consideration of or making a distinction in favor of or against a person based on group, class or category to which that person or patient belongs rather than on merit. Marginalization refers to the process whereby someone will be pushed to the edge of a group and accorded lesser importance. This is predominantly a social phenomenon by which a minority or subgroup or a group of people having same type of communicable disease is excluded and their needs or desires gets ignored. Vulnerable and marginalized populations bear an undue proportion of health problems. Overt or implicit discrimination violates one of the fundamental principles of human rights. It often lies at the root of poor health status and results in the lack of targeted policies and programmes and of access to services and other government structures relevant to health. Many factors can contribute to one's vulnerability to TB. Being poor, of a minority group, a migrant or refugee, a child, a prisoner, or having a weak immune system due to HIV or substance abuse are all factors that can make someone more likely to become sick with TB. Prejudice is an unfavorable opinion or feeling formed beforehand or without proper knowledge, thought and reason. All the unreasonable feelings, opinions, attitudes especially of a hostile nature, regarding an ethnic, racial, social or religious group will be considered prejudice. In today's scientific world attention has been recently given to psychosocial problems associated with disabilities and communicable diseases. Health-related stigma has highlighted the need for better understanding of the structural influences on stigma processes [24-26]. Social relations of power, prejudice, discrimination and other structural dynamics in the production of stigma have gained currency in scholarly discourse [27-29]. However, few empirical studies have been conducted on the influence of social structure on health-related stigma. Stigma and prejudice are each complex concepts that encompass individual experience the interaction between non-marginalized and marginalized groups, and broader structural and social phenomena such as power relations, historical contingencies, community practices and program/policy design. Stigma research has traditionally emphasized studying people with "unusual" conditions such as facial disfigurement, HIV/AIDS, TB, short stature and mental illness. Progress has been made in explicating the pathways through which stigma and prejudice impact health in this young field of inquiry. However, conference participants agreed, much more work is needed to deepen our understanding of the many ways that stigma and prejudice affect marginalized persons leading to psychological, social and biological consequences. In general, stigma and prejudice are believed to adversely impact health through five pathways. First, studies have documented that interactions between marginalized and non-marginalized individuals that are perceived to be discriminatory are health harming because of the stress processes they activate [30]. Discriminatory interactions also have negative implications for health and well being as they can lead to mistreatment in educational settings, in finding jobs, housing and health care [31]. Second, many of the health disadvantages experienced by marginalized persons occur outside a model in which one person consciously does something unfair to another. There are numerous examples of how structural forms of stigma and prejudice such as segregation lead to poor health outcomes for marginalized individuals because they are denied access to basic health and life resources [32]. Third, unconscious forms of prejudice perpetrated by non-marginalized individuals are being shown to occur spontaneously, automatically and without the full awareness of the persons perpetrating this form of prejudice [33]. There is growing evidence that these unconscious biases are not only perceived by marginalized persons [34], but may lead to discriminatory behavior among persons who hold these unconscious biases [35]. Fourth, is the internalization of stigma and prejudice by marginalized individuals, which has been linked to serious health harming consequences ranging from constricted social networks [36], compromised quality of life [37, 38], depressive symptoms [39] and to unemployment and income loss [40]. Finally, stigma and prejudice researchers write about vigilance in the anticipation of negative treatment chronically activating psychological stress responses and leading to impaired social interactions between marginalized and non-marginalized persons [41]. Despite growing clarity about the linkages between stigma, prejudice and health, much more research is needed to understand these pathways fully. Meyer, Schwartz, and Frost (2008) measure multiple types of stress including: common forms of stress (e.g., stressful life events), and those uniquely experienced by persons of marginalized social statuses (in this case among persons of minority sexual orientation, race/ethnicity and gender). The diversity and depth of research work underscores the complexity of the linkages between stigma, prejudice and health. The development of practical interventions and policy to reduce and attenuate the impacts of stigma and prejudice on health for marginalized populations depends on more fully understanding these linkages. A critical review of the evolution of TB control programmes in India suggests that TB control programmes largely focused on diagnosing and treating TB disease only, and the human and social 
aspect of care received minimal attention. No effort is made to evaluate patients' social and psychological experiences under this programme. However, exploring psychosocial problems is particularly relevant in the case of TB, where patients and physicians have divergent perceptions, and concerns about TB care. According to Kleinman (1980) [42], to provide care that effectively meets the patients' and their family members' psychosocial needs, a culturally sensitive clinician not only focuses on disease and its treatment, but on patients' ideas about what is wrong with them, their fears about illness, and the impact illness has on their physical and psychosocial functioning. Review of literature suggests that there are a few qualitative studies on psychosocial experiences and feelings of rural TB patients upon learning that they were TB infected in the state of Andhra Pradesh. Understanding psychosocial problems is crucial for formulating policies, programmes and interventions that provide culturally sensitive supportive care to the patients. For instance, an awareness of psychosocial suffering experiences of patients enables the physician to personalize his/ her approach to patient care, and to motivate, inspire or communicate with patients more effectively.

\section{Conclusion}

Researchers have estimated that 2 billion people, one-third of the global population, are infected with Mycobacterium tuberculosis, the bacterium that causes TB [43]. Although initially formulated as an area for social science research, stigma, discrimination, marginalization and prejudice has now become an important topics for public health. These contribute to suffering, which may further impair health, and it interferes with appropriate use of health services, even when they are available. Goffman's conceptualization of stigma has guided a wide range of social research studies and we have suggested ways of rethinking the concept so that it may better guide public health research, policy and action. Our formulation is rooted in his gloss of stigma as disqualification from full social acceptance as its definitive feature. It applies this concept to social aspects of international health problems that are targets of stigma. Our review highlights the importance of accounting for particular features of stigmatized health problems and the value of identifying determinants and effects of practical significance for health policy. With reference to prior work in the field, researchers focused on psychosocial issues of Tuberculosis patients and try to contribute research area of International Public Health.

\section{Acknowledgements}

We sincerely acknowledge the valuable support from Mr. Showkat Ahmad Ganaie and Mr. Suhail Ahmad Bhat from Post Graduate Department of Psychology, University of Kashmir, Hazratbal, $\mathrm{J} \& \mathrm{~K}$.

\section{References}

[1]. World Health Organization (2008) Closing the gap in a generation: health equity through action on the social determinants of health: Commission on Social Determinants of Health final report. Commission on Social Determinants of Health, Geneva, Switzerland.

[2]. World Health Organization (2014) Global tuberculosis report 2014. Geneva: World Health Organization, 2014. http://www.who.int/tb/publications/ global_report/en/

[3]. World Health Organization (2010) Multidrug and Extensively Drug-Resistant TB (M/XDR-TB): 2010 Global Report on Surveillance and Response. http://www.who.int/tb/features_archive/m_xdrtb_facts/en/index.html
[4]. Central TB Division (2011) Official website of the Revised National TB Control Programme, Directorate General of Health Services, Ministry of Health \& Family Welfare, Government of India. www.tbcindia.org.

[5]. Crocker J, Major B, Steele C (1998) Social stigma. In: DT Gilbert, ST Fiske, G Lindzey (Eds). The handbook of social psychology. (4th edtn), Mc Graw Hill, New York. 2: 504-553.

[6]. Émile Durkheim (1982) Rules of Sociological Method (1895). The Free Press, W.D. Halls, New York.

[7]. Erving Goff man (1963) Stigma: Notes on the Management of Spoiled Identity. Prentice-Hall. ISBN 0-671-62244-7

[8]. Huy TQ, Johansson A, Long NH (2007) Reasons for not reporting deaths: a qualitative study in rural Vietnam. World Health Popul 9(1): 14-23.

[9]. Long NH, Johansson E, Diwan VK, Winkvist A (2001) Fear and social isolation as consequences of tuberculosis in Vietnam: a gender analysis. Health Policy 58(1): 69-81.

[10]. Johansson E, Diwan VK, Huong ND, Ahlberg BM (1996) Staff and patient attitudes to tuberculosis and compliance with treatment: an exploratory study in a district in Vietnam. Tuber Lung Dis 77(2): 178-83.

[11]. AhChing LP, Sapolu M, Samifua M, Yamada S (2001) Attitudes regarding tuberculosis among Samoans. Pac Health Dialog 8(1): 15-19.

[12]. Liefooghe R, Michiels N, Habib S, Moran MB, De Muynck A (1995) Perception and social consequences of tuberculosis: a focus group study of tuberculosis patients in Sialkot, Pakistan. Soc Sci Med 41(12): 1685-1692.

[13]. West EL, Gadkowski LB, Ostbye T, Piedrahita C, Stout JE (2008) Tuberculosis knowledge, attitudes, and beliefs among North Carolinians at increased risk of infection. N C Med J 69(1): 14-20.

[14]. Demissie M, Getahun H, Lindtjorn B (2003) Community tuberculosis care through "TB clubs" in rural North Ethiopia. Soc Sci Med 56(10): 2009_ 2018.

[15]. Kelly P (1999) Isolation and stigma: the experience of patients with active tuberculosis. J Community Health Nurs 16(4): 233-241.

[16]. Liefooghe R, Baliddawa JB, Kipruto EM, Vermeire C, De Munynck AO (1997) From their own perspective. A Kenyan community's perception of tuberculosis. Trop Med Int Health 2(8): 809-821.

[17]. Balasubramanian R, Garg R, Santha T, Gopi PG, Subramani R, et al. (2004) Gender disparities in tuberculosis: report from a rural DOTS programme in south India. Int J Tuberc Lung Dis 8(3): 323-332.

[18]. Eastwood SV, Hill PC (2004) A gender-focused qualitative study of barriers to accessing tuberculosis treatment in The Gambia, West Africa. Int J Tuberc Lung Dis 8(1): 70-75.

[19]. Sengupta S, Pungrassami P, Balthip Q, Strauss R, Kasetjaroen Y, et al. (2006) Social impact of tuberculosis in southern Thailand: views from patients, care providers and the community. Int J Tuberc Lung Dis 10(9): 1008-1012.

[20]. Johansson E, Long NH, Diwan VK, Winkvist A (2000) Gender and tuberculosis control: perspectives on health seeking behaviour among men and women in Vietnam. Health Policy 52(1): 33-51.

[21]. Baral SC, Karki DK, Newell JN (2007) Causes of stigma and discrimination associated with tuberculosis in Nepal: a qualitative study. BMC Public Health 7:211.

[22]. Dimitrova B, Balabanova D, Atun R, Drobniewski F, Levicheva V, et al. (2006) Health service providers perceptions of barriers to tuberculosis care in Russia. Health Policy Plan 21(4): 265-274.

[23]. UNAIDS (2000) HIV- and AIDS-related stigmatization, discrimination and denial: forms, contexts and determinants. Research studies from Uganda and India. UNAIDS, Geneva.

[24]. Pescosolido BA, Martin JK, Lang A, Olafsdottir S (2008) Rethinking theoretical approaches to stigma: A framework integrating normative influences on stigma (FINIS). Soc Sci Med 67(3): 431-440.

[25]. Phelan JC, Link BG, Dovidio JF (2008) Stigma and prejudice: One animal or two? Soc Sci Med 67(3): 358-367.

[26]. Scambler G (2006) Sociology, social structure and health-related stigma. Psychol Health Med 11(3): 288-295.

[27]. Link B, Castille DM, Stuber J (2008) Stigma and coercion in the context of outpatient treatment for people with mental illnesses. Soc Sci Med 67(3): 409-419.

[28]. Link BG, Phelan JC (2001) Conceptualizing stigma. Annual Review of Sociology 27: 363-385.

[29]. Weiss MG (2008) Stigma and the social burden of neglected tropical diseases. PLOS Negl Trop Dis 2(5): e237.

[30]. Krieger N (1990) Racial and gender discrimination: risk factors for high blood pressure? Soc Sci Med 30(12): 1273-1281.

[31]. Wahl OF (1999) Mental health consumers' experience of stigma. Schizophr Bull 25(3): 467-478.

[32]. LaVeist TA (2003) Racial segregation and longevity among African Americans: an individual-level analysis. Health Serv Res 38(6 Pt 2): 1719-1733.

[33]. Dovidio JF, Gaertner SL (1998) On the nature of contemporary prejudice: the causes, consequences, and challenges of aversive racism. In: Eberhordt J, Fiske ST, editors. Confronting racism: the problem and the response. New- 
bury Park, CA: Sage 3-32.

[34]. Richeson JA, Shelton JN (2005) Thin slices of racial bias. Journal of NonVerbal Behavior 29(1): 75-86.

[35]. Dovidio JF, Gaertner SL (2004) Aversive racism. In: Zanna MP, editor. San Diego, CA: Academic Press. Advances in experimental social psychology 36: $1-51$.

[36]. Link BG, Cullen FT, Struening E, Shrout P, Dohrenwend BP (1989) A modified labeling theory approach in the area of mental disorders: an empirical assessment. American Sociological Review 54(3): 400-423.

[37]. Rosenfield S (1997) Labeling and mental illness: the effects of received services and perceived stigma on life satisfaction. American Sociological Review. 62(4): 660-672.

[38]. Wright ER, Gronfein WP, Owens TJ (2000) Deinstitutionalization, social rejection and the self-esteem of former mental patients. J Health Soc Behav 41(1): 68-90.
[39]. Link BG, Struening EL, Rahav M, Phelan JC, Nuttbrock L (1997) On stigma and its consequences: evidence from a longitudinal study of men with dual diagnoses of mental illness and substance abuse. J Health Soc Behav 38(2): 177-190.

[40]. Link BG (1987) Understanding labeling effects in the area of mental disorders: an assessment of the effects of expectations of rejection. American Sociological Review 52(1): 96-112.

[41]. Meyer IH (2003b) Prejudice, social stress and mental health in lesbian, gay, and bisexual populations: conceptual issues and research evidence. Psychol Bull 129(5): 674-697.

[42]. Kleinman A (1980) Patients and Healers in the Context of Culture. University of California Press, London.

[43]. Tuberculosis: Major Barriers to Addressing the Growing Epidemic. http:// www.iom.edu/_/media/Files/Activity\%20Files/Research/DrugForum/ IOM_MDRTB_whitepaper_2009_01_14_FINAL_Edited.pdf 